\title{
Plasma Aminothiol Profile and Some of Its Determinants in Apparently Healthy Azorean Subjects
}

\author{
Ana Lima, Rita Ferin, José Baptista, and M. Leonor Pavão \\ Center of Research in Natural Resources (CIRN) and Department of Technological Sciences and Development, \\ University of the Azores, Ponta Delgada, 9501-801 Azores, Portugal \\ Correspondence should be addressed to José Baptista; baptista@uac.pt
}

Received 22 October 2013; Accepted 6 January 2014; Published 16 February 2014

Academic Editors: P. Leroy and N. Visavadiya

Copyright (C) 2014 Ana Lima et al. This is an open access article distributed under the Creative Commons Attribution License, which permits unrestricted use, distribution, and reproduction in any medium, provided the original work is properly cited.

Objectives. To evaluate the plasma aminothiol profile (PAP) and serum gamma-glutamyltransferase (GGT) activity, as well as plasma folate, vitamin $\mathrm{B}_{12}$, and vitamin $\mathrm{B}_{6}$ concentrations, in 326 apparently healthy subjects from the Azores archipelago (Portugal). Also eventual relationships of PAP with conventional risk factors for atherosclerosis were investigated, aiming at the finding of early blood markers of the disease. Design and Methods. This was an observational cross-sectional study, where participants were split into two groups: one with a normal and another with an altered PAP (at least one aminothiol out of its reference concentration range). Results. About $76 \%$ of subjects had an altered PAP, mainly due to low glutathione levels $(<1.5 \mu \mathrm{mol} / \mathrm{L})$, mostly associated with normal GGT activity. Prevalence of hyperhomocysteinemia was 10\%, where only $33 \%$ had some B-vitamin deficiency. The risk for atherosclerosis was more evidenced in subjects exhibiting both deficient GSH concentration and increased serum GGT activity. Conclusions. An altered PAP, namely, when caused by low GSH levels in the absence of alterations in the Hcy, or Cys, or Cys-Gly concentrations and in serum GGT activity, might reveal a subclinical stage of atherogenesis and should be explored as a potential early marker of atherosclerosis.

\section{Introduction}

Atherosclerosis (AT), the major origin of cardiovascular disease (CVD), is a chronic multifactorial condition which can develop as a silent and progressive disease [1], whose clinical symptoms arise in advanced stages of the pathology as irreversible or deadly. This fact justifies the need to identify early blood markers for this condition in asymptomatic populations. It is well known that oxidative processes play a crucial role in atherogenesis [2] and can contribute to disrupt redox homeostasis which is essential for maintaining normal cellular functions. In recent years, there has been an increasing interest in the measurement of aminothiols in human plasma, since disturbances in thiol homeostasis have been linked to many disorders, namely, CVD [3]. The major plasma aminothiols are homocysteine (Hcy), cysteine (Cys), cysteinylglycine (Cys-Gly), and glutathione (GSH), which serve numerous vital functions, including detoxification and regulation of cellular metabolism, enzymatic activity, protein synthesis and structure [4], protection of cellular components against oxidative stress [5], formation of bioactive molecules, and participation in amino acid transport, among others [6]. All these aminothiols interact via redox, namely, disulfide exchange reactions, and reduced, oxidized, and protein bound forms of these species comprise a dynamic system referred to as the redox thiol status [7]. Hcy is produced from methionine metabolism and can be remethylated to methionine by methionine synthase, which requires vitamin $B_{12}$ as a cofactor and 5-methyltetrahydrofolate as a substrate. Alternatively, $\mathrm{Hcy}$ can be transsulfurated to Cys by two sequential vitamin $\mathrm{B}_{6}$-dependent reactions [8]. Besides being an Hcy byproduct, Cys is a GSH precursor inside cells, which plays vital functions, namely, as antioxidant. The action of gamma-glutamyltransferase (GGT) on extracellular GSH produces glutamate and Cys-Gly, which is usually taken within intracellular milieu by membrane dipeptidases to form Cys and Gly as precursors for GSH resynthesis [9]. Serum GGT activity is conventionally interpreted as a marker of alcohol abuse and liver dysfunction, but also it has been shown to correlate with CVD [10]. Oxidative processes lead 
to depletion of GSH, which induces the expression of GGT, along with a subsequent elevation of its activity in serum [11]. Therefore, GGT may predict various diseases, such as CVD [12], as a marker of oxidative stress.

As far as we know no study has addressed the value of the plasma aminothiol profile (plasma total concentrations of Hcy, Cys, Cys-Gly, and GSH) as a potential criterion for subclinical atherosclerosis detection and its relationship with well-established cardiovascular risk factors for AT. Therefore, the main objective of this study was to evaluate the plasma aminothiol profile (PAP) and serum GGT activity, as well as plasma folate, vitamin $B_{12}$, and vitamin $B_{6}$ concentrations, in a group of apparently healthy subjects from the Azores archipelago (Portugal). Furthermore, their relationship with some conventional AT risk factors, such as gender, age, obesity, blood pressure, dyslipidemia, and smoking, was also considered, aiming the search for early blood markers of subclinical stages of the disease.

\section{Methods}

2.1. Subjects. The study group consisted of 326 (189 women and 137 men) volunteers aged 20-61 years, apparently healthy and asymptomatic for AT, all born and residing in the Azores (Portugal). Subjects with a history of CVD, diabetes mellitus, hepatic dysfunction, or any other chronic disease, as well as those under vitamin supplementation since the last two months were excluded. An additional exclusion criterion was the existence of serum triglyceride (TG) concentrations above $400 \mathrm{mg} / \mathrm{dL}$. Participants were requested to complete a questionnaire on their medical history, lifestyle, and medicine intake. Body weight and height were measured to calculate body mass index (BMI), as well as blood pressure (BP). The study received approval from the ethical committee of our institution and all participants provided written informed consent.

2.2. Study Design. This observational study had a crosssectional design where subjects were assigned into one of two groups: the normal PAP (nPAP) group respected subjects where all aminothiols concentrations were within the respective reference range, and the altered PAP (aPAP) group which was formed by those having at least one aminothiol out of its reference concentration range, as follows: Hcy $\geq$ $15 \mu \mathrm{mol} / \mathrm{L}$ [13] and/or Cys $>250 \mu \mathrm{mol} / \mathrm{L}$ [14] and/or CysGly $>36 \mu \mathrm{mol} / \mathrm{L}[15]$ and/or GSH $<1.5 \mu \mathrm{mol} / \mathrm{L}$. High serum GGT activity was defined as $>36 \mathrm{U} / \mathrm{L}$ for women and $>61 \mathrm{U} / \mathrm{L}$ for men (according to manufacturer's instruction). Plasma folate deficiency was defined for concentrations $\leq 4 \mathrm{ng} / \mathrm{mL}$; vitamin $\mathrm{B}_{6}$ or vitamin $\mathrm{B}_{12}$ deficiencies were considered for levels of pyridoxal- $5^{\prime}$-phosphate $(\mathrm{PLP})<20 \mathrm{nmol} / \mathrm{L}$ [16] or $\mathrm{B}_{12}<250 \mathrm{pg} / \mathrm{mL}$ [17], respectively.

Subjects exhibiting $\mathrm{BMI} \geq 30 \mathrm{Kg} / \mathrm{m}^{2}$ were classified as obese and those with $25<\mathrm{BMI}<30 \mathrm{Kg} / \mathrm{m}^{2}$ were overweight. Hypertension was settled for systolic BP $\geq 140 \mathrm{mmHg}$ and/or diastolic $\mathrm{BP} \geq 90 \mathrm{mmHg}$ and/or when taking antihypertensive medication. Hyperlipidemia was defined as serum total cholesterol (TC) concentration $\geq 200 \mathrm{mg} / \mathrm{dL}$, and/or TG levels $\geq 150 \mathrm{mg} / \mathrm{dL}$, and/or when under current lipid-lowering medication.

2.3. Sample Collection. A single fasting venous blood sample was collected from all subjects by a standardized procedure of venipucture. The blood was drawn into $4.9 \mathrm{~mL}$ heparinised Sarstedt vacuntainers (Sarstedt AG \& Co., Nümbrecht, Germany) and into $10 \mathrm{~mL}$ Sarstedt vacutainers without anticoagulant. After centrifugation at $1500 \times \mathrm{g}$ for $15 \mathrm{~min}$ at $4^{\circ} \mathrm{C}$ for serum and $2500 \times \mathrm{g}$ for $15 \mathrm{~min}$ at $4^{\circ} \mathrm{C}$ for plasma, both fractions were separated, divided into $200 \mu \mathrm{L}$ aliquots, and stored at $-80^{\circ} \mathrm{C}$ until further analysis. One aliquot of serum was immediately used to evaluate the lipid parameters and GGT activity.

2.4. Biochemical Analyses. Total (reduced, oxidized, and protein bound species) plasma concentrations of Hcy, Cys, CysGly, and GSH were measured by a RP-HPLC methodology with fluorescence detection, as described elsewhere [18]. PLP analysis by HPLC with fluorescence detection was performed according to the method of Kimura et al. [19]. Plasma folate and vitamin $\mathrm{B}_{12}$ levels were determined by using electrochemiluminescence competitive immunoassay kits on Cobas 6000 analyzer (Roche Diagnostics). Serum TC, HDL-cholesterol (HDL-C), and TG, as well as GGT activity, were measured by standardized enzymatic methods by using Roche diagnostic kits on a Cobas Integra 400 plus (Roche Diagnostics). LDL-cholesterol (LDL-C) was calculated using the Friedwald formula [20]. The intraassay coefficients of variation for all these biochemical parameters were accepted for values $\leq 7 \%$.

2.5. Statistical Analysis. The SPSS 17.0 software (Statistical Packages for Social Sciences, for Windows) was used for data evaluation. Results were expressed as mean \pm SD and a twotailed $P<0.05$ value was considered statistically significant. Because the variables were not normally distributed, the following nonparametric methods were used: chi-square test for categorical variables, Mann-Whitney $U$ test and KruskalWallis test (followed by Dunn test) in the case of independent samples and Spearman's rank correlation coefficients were calculated to test relationship between two variables.

\section{Results}

3.1. Baseline Characteristics of the Study Population. Table 1 shows the baseline characteristics of the study population by gender. Men, who represented $42 \%$ of all participants, were significantly younger $(8 \% ; P<0.05)$ and exhibited a higher prevalence of smokers than women (34\% versus $13 \%$; $P<$ 0.001). Approximately half of the subjects were hypertensive, namely, men. More than $70 \%$ of subjects had excessive weight, where the prevalence of obesity in women was significantly higher $(46 \% ; P<0.05)$ than in men.

The majority of subjects in both genders exhibited a moderate hyperlipidemia (according to the NCEP/ATP III classification [21]), mainly due to hypercholesterolemia. No sex-related differences were observed except in HDL-C, 
TABLE 1: Baseline characteristics of the study population, according to gender.

\begin{tabular}{|c|c|c|c|c|}
\hline Parameters & All (326) & Women (189) & Men (137) & $P$ \\
\hline Age (years) & $41 \pm 10$ & $42 \pm 10$ & $39 \pm 10$ & $<0.05$ \\
\hline Smoking (\%) & 22 & 13 & 34 & $<0.001$ \\
\hline \multicolumn{5}{|l|}{ Blood pressure (mmHg) } \\
\hline Systolic BP & $131 \pm 22$ & $128 \pm 23$ & $134 \pm 22$ & $<0.01$ \\
\hline Diastolic BP & $80 \pm 14$ & $78 \pm 13$ & $81 \pm 15$ & $<0.05$ \\
\hline Hypertension (\%) & 45 & 40 & 51 & $<0.05$ \\
\hline \multicolumn{5}{|l|}{$\operatorname{BMI}\left(\mathrm{Kg} / \mathrm{m}^{2}\right)$} \\
\hline Overweight (\%) & 39 & 33 & 48 & $<0.01$ \\
\hline Obese (\%) & 33 & 38 & 26 & $<0.05$ \\
\hline \multicolumn{5}{|l|}{ Serum lipids (mg/dL) } \\
\hline Triglycerides & $120 \pm 39$ & $114 \pm 57$ & $127 \pm 70$ & NS \\
\hline Total cholesterol & $207 \pm 38$ & $209 \pm 35$ & $204 \pm 41$ & NS \\
\hline HDL cholesterol & $59 \pm 15$ & $64 \pm 15$ & $53 \pm 13$ & $<0.001$ \\
\hline LDL cholesterol & $128 \pm 35$ & $126 \pm 35$ & $130 \pm 36$ & NS \\
\hline Hyperlipidemia (\%) & 65 & 66 & 64 & NS \\
\hline \multicolumn{5}{|l|}{ Plasma thiols $(\mu \mathrm{mol} / \mathrm{L})$} \\
\hline Hcy & $10 \pm 3$ & $9 \pm 3$ & $11 \pm 4$ & $<0.001$ \\
\hline Cys & $199 \pm 39$ & $196 \pm 39$ & $203 \pm 39$ & NS \\
\hline Cys-Gly & $32 \pm 6$ & $29 \pm 5$ & $35 \pm 6$ & $<0.001$ \\
\hline GSH & $1.4 \pm 0.6$ & $1.3 \pm 0.5$ & $1.6 \pm 0.6$ & $<0.001$ \\
\hline Hyperhomocysteinemia (\%) & 10 & 6 & 16 & $<0.01$ \\
\hline Hypercysteinemia (\%) & 9 & 7 & 13 & NS \\
\hline High Cys-Gly (\%) & 24 & 12 & 40 & $<0.001$ \\
\hline Low GSH (\%) & 57 & 63 & 47 & $<0.01$ \\
\hline Serum GGT activity (U/L) & $33 \pm 35$ & $24 \pm 27$ & $44 \pm 42$ & $<0.001$ \\
\hline High GGT activity (\%) & 15 & 13 & 17 & NS \\
\hline \multicolumn{5}{|l|}{ Plasma vitamins } \\
\hline Folate (ng/mL) & $8 \pm 3$ & $8 \pm 3$ & $8 \pm 3$ & NS \\
\hline Deficiency (\%) & 8 & 10 & 4 & NS \\
\hline Vitamin $B_{12}(\mathrm{pg} / \mathrm{mL})$ & $569 \pm 313$ & $567 \pm 359$ & $571 \pm 232$ & NS \\
\hline Deficiency (\%) & 6 & 9 & 1 & $<0.01$ \\
\hline Vitamin $B_{6}(\mathrm{nmol} / \mathrm{L})$ & $53 \pm 23$ & $48 \pm 26$ & $61 \pm 28$ & $<0.001$ \\
\hline Deficiency (\%) & 4 & 6 & 1 & $<0.05$ \\
\hline
\end{tabular}

Values are presented as mean $\pm \mathrm{SD}$, except otherwise indicated. Figures in parenthesis are the number of subjects $(n) . P$ values denote significant differences between genders. NS: not significant.

in which concentration was significantly higher $(21 \% ; P<$ 0.001 ) in women than in men, as expected.

Ten percent of all participants exhibited hyperhomocysteinemia (HHcy) but none had intermediate or severe HHcy (>30 $\mu \mathrm{mol} / \mathrm{L})$. However, more than half showed low GSH levels and almost one-fourth (mainly men) had high plasma Cys-Gly concentration.

The mean values of Hcy, Cys-Gly, GSH, and vitamin $\mathrm{B}_{6}$ concentrations, as well as of GGT activity, were significantly higher $(P<0.001)$ in men than in women, but no differences between genders were observed for Cys, folate, or vitamin $B_{12}$ concentrations.

A small number of subjects had deficient plasma folate, vitamin $\mathrm{B}_{12}$, or vitamin $\mathrm{B}_{6}$ levels. Furthermore, only $33 \%$ of the hyperhomocysteinemics had some B-vitamin deficiency, being folate the most frequent (27\%). In addition, negative associations between Hcy and folate or vitamin $B_{12}$
( $r=-0.45, P<0.001 ; r=-0.18, P<0.01$, resp. $)$ were found in the whole population, despite gender, but no such relationship was found for vitamin $\mathrm{B}_{6}$.

3.2. Analysis of Parameters, according to Plasma Aminothiol Profile. More than three-quarters of all subjects exhibited an altered PAP, as shown in Table 2, which was mainly due to the existence of very low GSH concentrations. Except for gender and for HDL-C (whose levels did not differ) all the other conventional risk factors (age, smoking, BP, BMI, and serum lipids) were highlighted in the aPAP group (Table 2).

Albeit at normal levels, serum GGT activity was significantly higher $(P<0.001)$ in the aPAP than in the nPAP group (in both genders). Moreover, the enzyme activity was positively associated with the concentrations of Hcy, Cys, or Cys-Gly $(r=0.28, P<0.001 ; r=0.23, P<0.001$; 
TABLE 2: Baseline characteristics of the study population, according to plasma aminothiol profile (PAP).

\begin{tabular}{|c|c|c|c|}
\hline Parameters & nPAP (79) & aPAP (247) & $P$ \\
\hline Sex, male (\%) & 42 & 42 & NS \\
\hline Age (years) & $36 \pm 10$ & $42 \pm 9$ & $<0.001$ \\
\hline Smoking (\%) & 19 & 23 & NS \\
\hline \multicolumn{4}{|l|}{ Blood pressure (mmHg) } \\
\hline Systolic BP & $126 \pm 21$ & $132 \pm 23$ & $<0.01$ \\
\hline Diastolic BP & $77 \pm 13$ & $80 \pm 14$ & NS \\
\hline Hypertension (\%) & 35 & 47 & NS \\
\hline \multicolumn{4}{|l|}{$\mathrm{BMI}\left(\mathrm{Kg} / \mathrm{m}^{2}\right)$} \\
\hline Overweight (\%) & 32 & 42 & NS \\
\hline Obese (\%) & 24 & 36 & NS \\
\hline \multicolumn{4}{|l|}{ Serum lipids (mg/dL) } \\
\hline Triglycerides & $101 \pm 48$ & $125 \pm 66$ & $<0.01$ \\
\hline Total cholesterol & $201 \pm 39$ & $209 \pm 37$ & $<0.05$ \\
\hline HDL cholesterol & $61 \pm 13$ & $59 \pm 15$ & NS \\
\hline LDL cholesterol & $123 \pm 35$ & $129 \pm 35$ & $<0.05$ \\
\hline Hyperlipidemia (\%) & 57 & 68 & NS \\
\hline Serum GGTactivity (U/L) & $19 \pm 13$ & $37 \pm 39$ & $<0.001$ \\
\hline \multicolumn{4}{|l|}{ Plasma vitamins } \\
\hline Folate (ng/mL) & $8 \pm 3$ & $8 \pm 3$ & NS \\
\hline Vitamin $B_{12}(\mathrm{pg} / \mathrm{mL})$ & $575 \pm 303$ & $567 \pm 316$ & NS \\
\hline Vitamin $\mathrm{B}_{6}(\mathrm{nmol} / \mathrm{L})$ & $54 \pm 24$ & $53 \pm 29$ & NS \\
\hline
\end{tabular}

nPAP: normal plasma aminothiol profile; aPAP: altered plasma aminothiol profile; NS: not significant. Values are presented as mean \pm SD, except otherwise indicated. Figures in parenthesis are the number of subjects $(n) . P$ values denote significant differences between groups.

$r=0.22, P<0.001$, resp. $)$ and negatively with GSH $(r=$ $-0.29, P<0.001)$ in the aPAP group, whilst in the nPAP-one only Cys-Gly correlated significantly with GGT. Also in the aPAP group, GGT activity was positively associated with TG concentration $(r=0.35, P<0.001)$.

The mean concentrations of all vitamins in plasma did not change with PAP (Table 2). In both nPAP and aPAP groups Cys was positively associated with TG $(r=0.23, P<0.05$; $r=0.19, P<0.01$, resp.). Furthermore, in the aPAP group, but not in the normal one, Cys was modestly, still significantly associated with LDL-C $(r=0.20, P<0.01)$ and was negatively correlated with HDL-C $(r=-0.22, P<0.001)$.

3.3. Analysis of Parameters in Subjects with an Altered PAP, according to the Concentrations of Aminothiols. Among aPAP subjects, 75\% exhibited low plasma GSH levels. Therefore, in order to get a more clear perception of the relationships among GSH and the other studied parameters, we looked, in more detail, at the concentrations of the various aminothiols in the aPAP subjects taking into account the respective reference range and split subjects into three subgroups (Table 3 ). The aPAP-1 subgroup was formed by individuals, where GSH was the only thiol exhibiting an altered concentration in plasma. The aPAP-2 included those who had at least one thiol in altered concentration except GSH, which was at normal levels. The remaining subjects (aPAP-3) had one or more thiols (including GSH) in altered concentration.

A dramatic change in gender prevalence was observed among subgroups. In fact, women seemed to be significantly more prone to exhibit low GSH levels, while men were more likely to present alterations in the other thiols concentrations. The average age was also significantly higher in aPAP-1 than in aPAP-2 subjects. The highest number of smokers was registered in the aPAP-3 subgroup. On the contrary, the prevalence of hypertension and hyperlipidemia as well as of mean BP and serum lipids profile did not differ among groups.

Concerning BMI, the presence of obese was prevalent in the subgroup where GSH was the only thiol at altered concentration. On the other hand, overweight subjects were significantly much less represented in the aPAP-1 than in both aPAP-2 and aPAP-3 subgroups (which did not differ). GGT activity was significantly increased $(P<0.001)$ in aPAP-3 as compared to that found in the other two subgroups where no differences in the activity were observed. Folate was the sole vitamin to be significantly decreased $(P<0.05)$ in both aPAP-2 and -3 subgroups but not in the aPAP-1 one, where it was at normal levels.

3.4. Analysis of Parameters in Subjects with Low GSH Levels, according to the Activity of GGT. Among aPAP-1 subjects, only $17 \%$ exhibited an increased GGT activity as compared to reference values (Table 4). In aPAP subjects where GSH was the only thiol at altered concentration, we further examined the aPAP-1 individuals, according to their GGT activity (Table 4). Those with a normal GGT activity were included in the nGGT subgroup, and those with high GGT activity formed the hGGT one. Women were mostly represented in 
TABLE 3: Conventional risk factors in subjects with an aPAP, split by 3 subgroups.

\begin{tabular}{|c|c|c|c|}
\hline \multirow{2}{*}{ Parameters } & \multicolumn{3}{|c|}{ aPAP } \\
\hline & aPAP-1 (129) & aPAP-2 (62) & aPAP-3 (56) \\
\hline Sex, male (\%) & 22 & $63^{*}$ & $66^{\dagger}$ \\
\hline Age (years) & $44 \pm 9$ & $39 \pm 10^{*}$ & $41 \pm 8$ \\
\hline Smoking (\%) & 16 & 23 & $37^{\dagger}$ \\
\hline \multicolumn{4}{|l|}{ Blood pressure (mmHg) } \\
\hline Systolic BP & $134 \pm 25$ & $128 \pm 20$ & $131 \pm 19$ \\
\hline Diastolic BP & $81 \pm 15$ & $79 \pm 12$ & $81 \pm 13$ \\
\hline Hypertension (\%) & 47 & 42 & 55 \\
\hline \multicolumn{4}{|l|}{$\mathrm{BMI}\left(\mathrm{Kg} / \mathrm{m}^{2}\right)$} \\
\hline Overweight (\%) & 34 & $53^{*}$ & 46 \\
\hline Obese (\%) & 43 & $24^{*}$ & 30 \\
\hline \multicolumn{4}{|l|}{ Serum lipids (mg/dL) } \\
\hline Triglycerides & $123 \pm 63$ & $127 \pm 75$ & $129 \pm 63$ \\
\hline Total cholesterol & $211 \pm 35$ & $208 \pm 41$ & $206 \pm 37$ \\
\hline HDL cholesterol & $61 \pm 15$ & $56 \pm 15$ & $56 \pm 16$ \\
\hline LDL cholesterol & $128 \pm 33$ & $133 \pm 40$ & $128 \pm 36$ \\
\hline Hyperlipidemia (\%) & 71 & 69 & 61 \\
\hline Serum GGT activity (U/L) & $33 \pm 34$ & $26 \pm 15$ & $59 \pm 57^{\dagger \ddagger}$ \\
\hline \multicolumn{4}{|l|}{ Plasma vitamins } \\
\hline Folate $(\mathrm{ng} / \mathrm{mL})$ & $9 \pm 4$ & $7 \pm 2^{*}$ & $7 \pm 2^{\dagger}$ \\
\hline Vitamin $B_{12}(p g / m L)$ & $604 \pm 360$ & $553 \pm 226$ & $494 \pm 280$ \\
\hline Vitamin $\mathrm{B}_{6}(\mathrm{nmol} / \mathrm{L})$ & $56 \pm 33$ & $53 \pm 27$ & $47 \pm 19$ \\
\hline
\end{tabular}

aPAP-1: only low GSH; aPAP-2: at least one aminothiol (Hcy, Cys, or Cys-Gly) elevated and normal GSH; aPAP-3: at least one aminothiol (Hcy, Cys, or Cys-Gly) elevated and low GSH; NS: not significant. Values are presented as mean \pm SD, except otherwise indicated. Figures in parenthesis are the number of subjects $(n)$.

${ }^{*} P<0.05$ when comparing aPAP-1 and aPAP-2 groups.

${ }^{\dagger} P<0.05$ when comparing aPAP-1 and aPAP-3 groups.

${ }^{\ddagger} P<0.05$ when comparing aPAP-2 and aPAP-3 groups.

the nGGT group, but the mean age of subjects did not differ between the two subgroups. Concerning BP, no significant differences were observed between them but a trend to hyper systolic BP was clearly patent in the hGGT one. The prevalence of both overweight and obesity did not differ with GGT activity.

Hyperlipidemia seemed more frequent in hGGT subjects than in those with nGGT activity, not only because of TC levels but because of TG. Moreover, both TC and LDL-C were not statistically different between the two subgroups while the levels of HDL-C were significantly decreased in the hGGT group.

Plasma GSH concentration reached its lowest mean value in hGGT subjects where both the levels of Hcy and Cys (but not of Cys-Gly) were significantly higher $(P<0.01)$ than in the nGGT subgroup, despite being within the respective reference range.

\section{Discussion}

As far as we know this is the first study where the main low molecular weight plasma aminothiols are considered together to define a normal or an altered PAP in asymptomatic subjects as a strategy to investigate early blood markers for atherosclerosis. The population in this study was formed by individuals with no recognized chronic diseases, including CVD and diabetes. In addition, a reasonably small group of participants were tobacco consumers, which diminishes potential confounding effects of smoking on the other evaluated parameters. Under those circumstances, it was not expectable to find at a great extent some of the conventional risk factors for AT. However, a high prevalence of hypertension and of hyperlipidemia (though modest) was detected, and excessive weight (overweight or obesity) was frequently observed.

4.1. Plasma Aminothiol Profile of the Study Population. When splitting our sample according to PAP (Table 2), the majority of subjects showed an altered profile. GSH was the major responsible for that, since the tripeptide was present at very low concentrations, with almost $60 \%$ of all subjects having plasma GSH levels $<1.5 \mu \mathrm{mol} / \mathrm{L}$. Moreover, some authors [22] take $2 \mu \mathrm{mol} / \mathrm{L}$ as the lower limit of its reference range, which if adopted would increase that amount to $82 \%$. The mean GSH levels found in the aPAP group were similar to those reported by Dhawan et al. [23] in coronary artery disease patients. However, total plasma GSH concentrations found in both CVD patients and controls by Shimizu and coworkers [24] were higher and within the reference range. 
TABLE 4: Conventional risk factors in aPAP subjects with only low GSH levels (aPAP-1), split by GGT activity.

\begin{tabular}{|c|c|c|c|}
\hline \multirow{2}{*}{ Parameters } & \multicolumn{2}{|c|}{ aPAP-1 (129) } & \multirow{2}{*}{$P$} \\
\hline & nGGT (107) & hGGT (22) & \\
\hline Sex, male (\%) & 18 & 41 & $<0.05$ \\
\hline Age (years) & $43 \pm 10$ & $46 \pm 8$ & NS \\
\hline Smoking (\%) & 17 & 14 & NS \\
\hline \multicolumn{4}{|l|}{ Blood pressure (mmHg) } \\
\hline Systolic BP & $132 \pm 24$ & $143 \pm 29$ & NS \\
\hline Diastolic BP & $80 \pm 14$ & $86 \pm 19$ & NS \\
\hline Hypertension (\%) & 46 & 50 & NS \\
\hline \multicolumn{4}{|l|}{$\operatorname{BMI}\left(\mathrm{Kg} / \mathrm{m}^{2}\right)$} \\
\hline Overweight (\%) & 33 & 41 & NS \\
\hline Obese (\%) & 43 & 45 & NS \\
\hline \multicolumn{4}{|l|}{ Serum lipids (mg/dL) } \\
\hline Triglycerides & $118 \pm 61$ & $149 \pm 70$ & $<0.05$ \\
\hline Total cholesterol & $212 \pm 37$ & $204 \pm 25$ & NS \\
\hline HDL cholesterol & $63 \pm 15$ & $53 \pm 11$ & $<0.01$ \\
\hline LDL cholesterol & $128 \pm 35$ & $126 \pm 23$ & NS \\
\hline Hyperlipidemia (\%) & 68 & 82 & NS \\
\hline \multicolumn{4}{|l|}{ Plasma thiols $(\mu \mathrm{mol} / \mathrm{L})$} \\
\hline Hcy & $8 \pm 2$ & $10 \pm 2$ & $<0.01$ \\
\hline Cys & $183 \pm 32$ & $205 \pm 23$ & $<0.01$ \\
\hline Cys-Gly & $28 \pm 4$ & $28 \pm 4$ & NS \\
\hline GSH & $1.1 \pm 0.2$ & $0.9 \pm 0.2$ & $<0.001$ \\
\hline \multicolumn{4}{|l|}{ Plasma vitamins } \\
\hline Folate (ng/mL) & $9 \pm 4$ & $8 \pm 3$ & NS \\
\hline Vitamin $B_{12}(\mathrm{pg} / \mathrm{mL})$ & $608 \pm 381$ & $581 \pm 241$ & NS \\
\hline Vitamin $B_{6}(\mathrm{nmol} / \mathrm{L})$ & $55 \pm 33$ & $57 \pm 30$ & NS \\
\hline
\end{tabular}

nGGT: normal GGT activity; hGGT: high GGT activity; NS: not significant. Values are presented as mean \pm SD, except otherwise indicated. Figures in parenthesis are the number of subjects $(n) . P$ values denote significant differences between groups.

This discrepancy reflects the complexity of GSH metabolism, involving multiple relationships with other molecules.

Women were more affected by low plasma GSH levels than men. A similar gender-related difference, which is not fully understood, was also reported by other authors [25]. On the other hand, mean Cys-Gly levels were within the normal range in this population. However, a relevant number of subjects with high Cys-Gly concentration have also contributed to form the aPAP group. In agreement with others $[12,26]$, men exhibited higher plasma Cys-Gly levels than women in both groups.

Elevated plasma Hcy or Cys concentrations also contributed, though in a smaller extent than the other referred aminothiols, to the size of the aPAP group. Men also had higher plasma Hcy and Cys levels than women (more visible in aPAP subjects), as usually observed [26]. This has been interpreted by the effect of estrogens in women because that difference disappears rapidly after menopause [27].

4.2. Aminothiols and GGT Activity. It is generally considered that GGT activity is higher in males than in females and that it constitutes the main regulator of GSH circulating concentrations [28], since its action results in GSH cleavage to Cys-Gly and then to Cys. Therefore a negative correlation between the two parameters, as observed, was expected. However, in this study group, only less than one-quarter of individuals with low GSH levels exhibited as well a serum GGT activity above normal values. This points out to other possible reasons underlying the occurrence of low plasma GSH levels: a decreased GSH synthesis inside cells; a deficiency on GSH efflux by GSH transporters; and/or a large utilization of GSH by cells, namely, in antioxidant defense. Moreover, GSH synthesis is rate-limited by the availability of Cys and a decrease in the Hcy transsulfuration pathway can also lead to GSH deficiency [29]. In fact, there are other routes for the production of Cys. Therefore, the normal mean plasma Cys levels found in this study also could result from the contribution of other processes, such as protein degradation, and not exclusively from degradation of GSH. Measurement of intracellular Cys and GSH concentrations would help to clarify this point.

GGT activity was more elevated in the aPAP group, perhaps because this enzyme activity is higher in perturbed metabolism of thiol compounds, as already seen by Giral et al. in metabolic syndrome [28] and in older subjects [30].

Besides correlating with GSH, GGT activity was also associated with the other aminothiols in the aPAP group 
(Table 3), but not in the normal one (except for Cys-Gly). This suggests that an altered PAP, whatever its cause, is indicative of oxidative stress. In particular, since GGT is regarded as a marker of that condition $[12,31]$, it is conceivable that oxidative damage generated by Hcy may elevate that enzyme activity [32] thus lowering plasma GSH levels. However, as stated before, only $10 \%$ of the study population had HHcy, yet moderate, and just a small part of subjects with low plasma GSH levels had high GGT activity as well. Therefore the possible contribution of HHcy to deficient GSH levels in the present study group is more likely to be rather limited.

\subsection{Hyperhomocysteinemia and B-Vitamins. As expected, an} inverse relationship was observed between Hcy and folate or vitamin $B_{12}$ concentration, as these vitamins are essential in Hcy metabolism. This is why they are the first therapeutic targets in the treatment of HHcy. However, in the present study, only $33 \%$ of subjects with HHcy had B-vitamin deficiencies. These results seem to confirm that Hcy metabolic pathways are very complex and under the control of many factors, both genetic and nongenetic [33].

A low prevalence of B-vitamin deficiencies was observed in the present study group, maybe due to the relatively young age of subjects, as those deficiencies usually enhance with age [34]. Concerning folate and vitamin $\mathrm{B}_{12}$ concentrations, no gender-related differences were found in this study, which is in accordance with data reported by Castro et al. [35] and Cascalheira et al. [36] in other Portuguese populations. On the contrary, men had higher vitamin $\mathrm{B}_{6}$ levels than women, strongly suggesting that estrogens play a role on this vitamin concentration in plasma [16].

4.4. Plasma Aminothiol Profile and BMI. The percentage of subjects with normal BMI was dramatically decreased in aPAP subjects, but both overweight and obesity prevalence did not differ significantly with plasma aminothiol profile. However, a fairly different situation was observed when considering, de per se, subjects with deficient GSH levels (Table 3), suggesting that obesity is much more associated with a decrease in GSH levels than with changes in the other thiols concentrations. Furthermore, the prevalence of both overweight and obesity in this study group did not seem to be particularly linked to GGT activity, since no differences were observed between n- and hGGT groups (Table 4), which is not in agreement with other reports [37].

\subsection{Plasma Aminothiol Profile, Its Determinants, and Lipid} Profile. Although apparently healthy, this study population revealed an important prevalence of hyperlipidemia, namely, hypercholesterolemia, which is a well-known risk factor for the development of AT and vascular diseases. As expected, women had higher levels of HDL-C and lower levels of TG than men, which may confer to them a lower risk of atherosclerosis as compared to men.

Subjects in the nPAP group had a better lipid profile and were younger than those in the altered one. Moreover, our data suggest that an aPAP is associated with high TG concentrations, especially in men, independently of age.
Unsurprisingly, Hcy did not correlate with lipid profile, thus confirming that $\mathrm{HHcy}$ is an independent risk factor for vascular diseases [38].

The correlation found between Cys and the lipid profile could reflect a prooxidant state, where Cys is readily oxidizable, giving rise to the production of free radical species [28], thereby promoting oxidative damage of LDL and facilitating foam cell formation [39]. Our results seem to be in accordance with those reported by Van den Brandhof et al. [40], who hypothesized that Cys levels may predict a highrisk profile for CVD, namely, in hyperlipidemics, as a marker of oxidative stress.

In this study, the prevalence of hyperlipidemia apparently did not differ with plasma aminothiol profile (Tables 2 and 3). However, among aPAP subjects, those exhibiting low GSH levels (aPAP-1) were also those where the prevalence of hyperlipidemia was significantly higher than that registered in normal PAP individuals, which reflects mainly the increased TC concentration observed. Furthermore, when looking at HDL-C, which average did not differ between nPAP and aPAP-1 subjects, a trend to a decreased value was detected in subjects where the concentration of some thiol(s), other than GSH, was changed.

Finally, the finding of altered levels of both TG and LDL-C in subjects with high GGT activity point out to the existence of two categories of subjects with low GSH levels-those who exhibit some oxidative stress (hGGT) and those, in a much larger amount (nGGT) who, albeit with deficient GSH levels, are still at a more protected situation regarding oxidative stress, as reflected by both their decreased TG concentrations and high HDL-C. If this is the case, the high levels of TC observed could rather reflect more their high HDL-C levels than those of LDL-C. As a consequence, LDL-C instead of TC seems to be a more adequate parameter to take into account when defining atherogenic dyslipidemia, as considered in recent publications [41].

\section{Conclusions}

Although apparently healthy and asymptomatic for AT, most participants in this study had an altered plasma aminothiol profile, which favors oxidative stress and atherogenesis. More than half aPAP subjects (namely, women), where most conventional risk factors for AT were more evidenced than in nPAP, revealed to be highly deficient in GSH but not in the other plasma aminothiols. Surprisingly, only a small part of plasma GSH deficient subjects showed simultaneously an increased serum GGT activity (hGGT), which is considered as a marker of oxidative stress. These seem to constitute the subgroup where the risk of AT is the highest within the study population, since both mean systolic BP and plasma TG levels reached the respective upper borderline of normality and HDL-C exhibited the lowest values (albeit within its reference range). GSH deficient subjects with normal GGT activity (nGGT) did not show any decrease in HDL-C as compared to nPAP subjects, appearing to be at a smaller, yet evidenced, risk than the former subgroup. 
This work suggests that an altered PAP, even when caused by low GSH levels in the absence of alterations in the Hcy, or Cys or Cys-Gly concentrations and in serum GGT activity, might reveal a subclinical stage of the atherogenic process. It also should be explored as a potential early blood marker of AT.

The underlying cause for having low GSH levels at a great extension in Azorean healthy subjects has to be investigated in future studies.

\section{Conflict of Interests}

The authors declare that there is no conflict of interests regarding the publication of this paper.

\section{Acknowledgments}

The authors are grateful to Jorge Morgado (Health Centre of Nordeste), Jorge Oliveira (Health Centre of Povoação), Emiliana Dias (Health Centre of Sta Cruz das Flores), Joaquim Ferreira da Silva (Health Centre of Sta Cruz da Graciosa), José Aires Raposo (Health Centre of Ribeira Grande), César Gonçalves (Health Centre of São Jorge), and all the technical staff for their support in blood collection and in the determination of serum lipid profile. This work was supported by the project "Search for nuclear and mitochondrial biomarkers of atherosclerosis in isolated populations from Azores Islands" with a reference M2.1.2/F/009/2007, funded by the Regional Government of the Azores and "High Prevalence pathologies in the Azores: Genomic and biochemical markers", funded by DRCT (M2.1.2/I/026/2008). Ana Lima was supported by a FRCT Grant M3.1.6/F/025/2009. Rita Ferin was a Ph.D. grantee of FCT [SFRH/BD/34322/2007].

\section{References}

[1] M. Noël, E. Dewailly, M. L. Chateau-Degat, É. Counil, E. A. Laouan-Sidi, and E. Lonn, "Cardiovascular risk factors and subclinical atherosclerosis among Nunavik Inuit," Atherosclerosis, vol. 221, no. 2, pp. 558-564, 2012.

[2] C. Ceconi, A. Boraso, A. Cargnoni, and R. Ferrari, "Oxidative stress in cardiovascular disease: myth or fact?" Archives of Biochemistry and Biophysics, vol. 420, no. 2, pp. 217-221, 2003.

[3] Y. Özkan, E. Özkan, and B. Imşek, "Plasma total homocysteine and cysteine levels as cardiovascular risk factors in coronary heart disease," International Journal of Cardiology, vol. 82, no. 3, pp. 269-277, 2002.

[4] M. E. McMenamin, J. Himmelfarb, and T. D. Nolin, "Simultaneous analysis of multiple aminothiols in human plasma by high performance liquid chromatography with fluorescence detection," Journal of Chromatography B, vol. 877, no. 28, pp. 3274-3281, 2009.

[5] E. Bald, G. Chwatko, R. Głowacki, and K. Kuśmierek, "Analysis of plasma thiols by high-performance liquid chromatography with ultraviolet detection," Journal of Chromatography A, vol. 1032, no. 1-2, pp. 109-115, 2004.

[6] E. Camera and M. Picardo, "Analytical methods to investigate glutathione and related compounds in biological and pathological processes," Journal of Chromatography B, vol. 781, no. 1-2, pp. 181-206, 2002.
[7] G. Atmaca, "Antioxidant effects of sulfur-containing amino acids," Yonsei Medical Journal, vol. 45, no. 5, pp. 776-788, 2004.

[8] J. Selhub, "Public health significance of elevated homocysteine," Food and Nutrition Bulletin, vol. 29, no. 2, pp. S116-S125, 2008.

[9] H. J. Forman, H. Zhang, and A. Rinna, "Glutathione: overview of its protective roles, measurement, and biosynthesis," Molecular Aspects of Medicine, vol. 30, no. 1-2, pp. 1-12, 2009.

[10] D. Drogan, C. Weikert, J. Dierkes et al., "Plasma $\gamma$-glutamyltransferase, cysteinyl-glycine, and oxidized low-density lipoprotein: a pathway associated with myocardial infarction risk?" Arteriosclerosis, Thrombosis, and Vascular Biology, vol. 30, no. 10, pp. 2053-2058, 2010.

[11] O. Turgut, A. Yilmaz, K. Yalta, F. Karadas, and M. Birhan Yilmaz, " $\gamma$-Glutamyltransferase is a promising biomarker for cardiovascular risk," Medical Hypotheses, vol. 67, no. 5, pp. 10601064, 2006.

[12] D. Drogan, C. Weikert, J. Dierkes et al., "Plasma $\gamma$-glutamyltransferase, cysteinyl-glycine, and oxidized low-density lipoprotein: a pathway associated with myocardial infarction risk?" Arteriosclerosis, Thrombosis, and Vascular Biology, vol. 30, no. 10, pp. 2053-2058, 2010.

[13] S. Kaul, A. A. Zadeh, and P. K. Shah, "Homocysteine hypothesis for atherothrombotic cardiovascular disease. Not validated," Journal of the American College of Cardiology, vol. 48, no. 5, pp. 914-923, 2006.

[14] P. M. Ueland, M. A. Mansoor, A. B. Guttormsen et al., "Reduced, oxidized and protein-bound forms of homocysteine and other aminothiols in plasma comprise the redox thiol status-a possible element of the extracellular antioxidant defense system," Journal of Nutrition, vol. 126, no. 4, pp. 1281S-1284S, 1996.

[15] L. Turell, S. Carballal, H. Botti, R. Radi, and B. Alvarez, "Oxidation of the albumin thiol to sulfenic acid and its implications in the intravascular compartment," Brazilian Journal of Medical and Biological Research, vol. 42, no. 4, pp. 305-311, 2009.

[16] M. S. Morris, M. F. Picciano, P. F. Jacques, and J. Selhub, "Plasma pyridoxal $5^{\prime}$-phosphate in the US population: the National Health and Nutrition Examination Survey, 2003-2004," The American Journal of Clinical Nutrition, vol. 87, no. 5, pp. 14461454, 2008.

[17] Institute of Medicine Food and Nutrition Board, Dietary Reference Intakes for Thiamin, Riboflavin, Niacin, Vitamin B6, Folate, Vitamin B12, Pantothenic Acid, Biotin, and Choline, National Academies Press, 1998.

[18] R. Ferin, M. L. Pavão, and J. Baptista, "Methodology for a rapid and simultaneous determination of total cysteine, homocysteine, cysteinylglycine and glutathione in plasma by isocratic RP-HPLC, Journal of Chromatography B, vol. 911, pp. 15-20, 2012.

[19] M. Kimura, K. Kanehira, and K. Yokoi, "Highly sensitive and simple liquid chromatographic determination in plasma of B6 vitamers, especially pyridoxal 5 '-phosphate," Journal of Chromatography A, vol. 722, no. 1-2, pp. 295-301, 1996.

[20] W. T. Friedewald, R. I. Levy, and D. S. Fredrickson, "Estimation of the concentration of low-density lipoprotein cholesterol in plasma, without use of the preparative ultracentrifuge," Clinical Chemistry, vol. 18, no. 6, pp. 499-502, 1972.

[21] Expert Panel on Detection, Evaluation, and Treatment of High Blood Cholesterol in Adults, "Executive summary of the third report of the National Cholesterol Education Program (NCEP) expert panel on detection, evaluation, and treatment of high blood cholesterol in adults (adult treatment panel III)," Journal 
of the American Medical Association, vol. 285, no. 19, pp. 24862497, 2001.

[22] S. E. Moriarty-Craige and D. P. Jones, "Extracellular thiols and thiol/disulfide redox in metabolism," Annual Review of Nutrition, vol. 24, pp. 481-509, 2004.

[23] S. S. Dhawan, P. Eshtehardi, M. C. McDaniel et al., "The role of plasma aminothiols in the prediction of coronary microvascular dysfunction and plaque vulnerability," Atherosclerosis, vol. 219, no. 1, pp. 266-272, 2011.

[24] H. Shimizu, Y. Kiyohara, I. Kato et al., "Relationship between plasma glutathione levels and cardiovascular disease in a defined population: the Hisayama study," Stroke, vol. 35, no. 9, pp. 2072-2077, 2004.

[25] A. Pastore, G. Federici, E. Bertini, and F. Piemonte, "Analysis of glutathione: implication in redox and detoxification," Clinica Chimica Acta, vol. 333, no. 1-2, pp. 19-39, 2003.

[26] D. W. Jacobsen, V. J. Gatautis, R. Green et al., "Rapid HPLC determination of total homocysteine and other thiols in serum and plasma: sex differences and correlation with cobalamin and folate concentrations in healthy subjects," Clinical Chemistry, vol. 40, no. 6, pp. 873-881, 1994.

[27] O. Stanger, W. Herrmann, K. Pietrzik et al., "Clinical use and rational management of homocysteine, folic acid, and B vitamins in cardiovascular and thrombotic diseases," Zeitschrift fur Kardiologie, vol. 93, no. 6, pp. 439-453, 2004.

[28] P. Giral, N. Jacob, C. Dourmap et al., "Elevated gammaglutamyltransferase activity and perturbed thiol profile are associated with features of metabolic syndrome," Arteriosclerosis, Thrombosis, and Vascular Biology, vol. 28, no. 3, pp. 587-593, 2008.

[29] E. Mosharov, M. R. Cranford, and R. Banerjee, "The quantitatively important relationship between homocysteine metabolism and glutathione synthesis by the transsulfuration pathway and its regulation by redox changes," Biochemistry, vol. 39, no. 42, pp. 13005-13011, 2000.

[30] D. H. Lee and D. R. Jacobs Jr., "Is serum gamma-glutamyltransferase a marker of exposure to various environmental pollutants?" Free Radical Research, vol. 43, no. 6, pp. 533-537, 2009.

[31] D. H. Lee, R. Blomhoff, and D. R. Jacobs Jr., "Is serum gamma glutamyltransferase a marker of oxidative stress?” Free Radical Research, vol. 38, no. 6, pp. 535-539, 2004.

[32] H. Sakuta, T. Suzuki, and T. Ito, "Serum $\gamma$-glutamyl transferase is associated with plasma total homocysteine in Japanese patients with type 2 diabetes," Acta Diabetologica, vol. 44, no. 4, pp. 177180, 2007.

[33] R. Castro, M. Barroso, M. Rocha et al., "The TCN2 776C>G polymorphism correlates with vitamin B12 cellular delivery in healthy adult populations," Clinical Biochemistry, vol. 43, no. 78, pp. 645-649, 2010.

[34] R. Clarke, J. Grimley Evans, J. Schneede et al., "Vitamin B12 and folate deficiency in later life," Age and Ageing, vol. 33, no. 1, pp. 34-41, 2004.

[35] R. Castro, I. Rivera, P. Ravasco et al., "5,10-methylenetetrahydrofolate reductase $677 \mathrm{C} \rightarrow \mathrm{T}$ and $1298 \mathrm{~A} \rightarrow \mathrm{C}$ mutations are genetic determinants of elevated homocysteine," Monthly Journal of the Association of Physicians, vol. 96, no. 4, pp. 297303, 2003.

[36] J. F. Cascalheira, M. C. Parreira, A. N. Viegas, M. C. Faria, and F. C. Domingues, "Serum homocysteine: relationship with circulating levels of cortisol and ascorbate," Annals of Nutrition and Metabolism, vol. 53, no. 1, pp. 67-74, 2008.
[37] H. S. Sang, S. K. Ihm, J. K. Yun et al., "Can $\gamma$-glutamyltransferase be an additional marker of arterial stiffness?" Circulation Journal, vol. 71, no. 11, pp. 1715-1720, 2007.

[38] S. Lussier-Cacan, M. Xhignesse, A. Piolot, J. Selhub, J. Davignon, and J. Genest Jr., "Plasma total homocysteine in healthy subjects: sex-specific relation with biological traits," The American Journal of Clinical Nutrition, vol. 64, no. 4, pp. 587-593, 1996.

[39] N. Jacob, E. Bruckert, P. Giral, M. J. Foglietti, and G. Turpin, "Cysteine is a cardiovascular risk factor in hyperlipidemic patients," Atherosclerosis, vol. 146, no. 1, pp. 53-59, 1999.

[40] W. E. Van den Brandhof, K. Haks, E. G. Schouten, and P. Verhoef, "The relation between plasma cysteine, plasma homocysteine and coronary atherosclerosis," Atherosclerosis, vol. 157, no. 2, pp. 403-409, 2001.

[41] A. Zeljkovic, J. Vekic, V. Spasojevic-Kalimanovska et al., "LDL and HDL subclasses in acute ischemic stroke: prediction of risk and short-term mortality," Atherosclerosis, vol. 210, no. 2, pp. 548-554, 2010. 


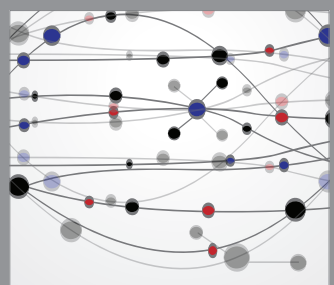

The Scientific World Journal
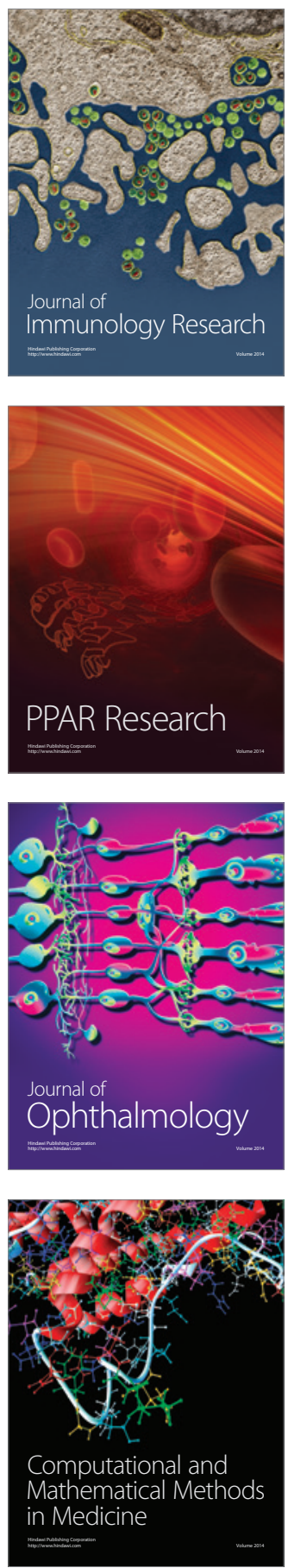

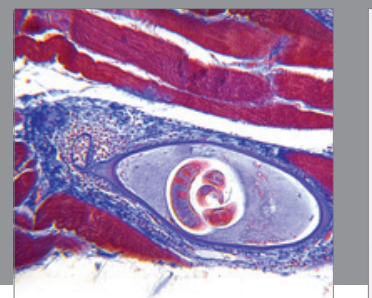

Gastroenterology

Research and Practice
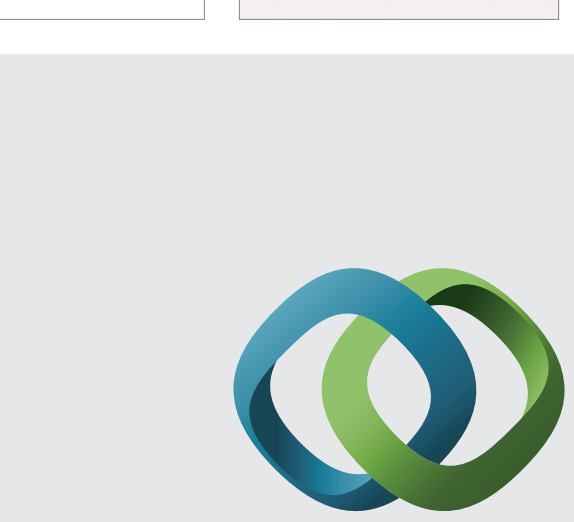

\section{Hindawi}

Submit your manuscripts at

http://www.hindawi.com
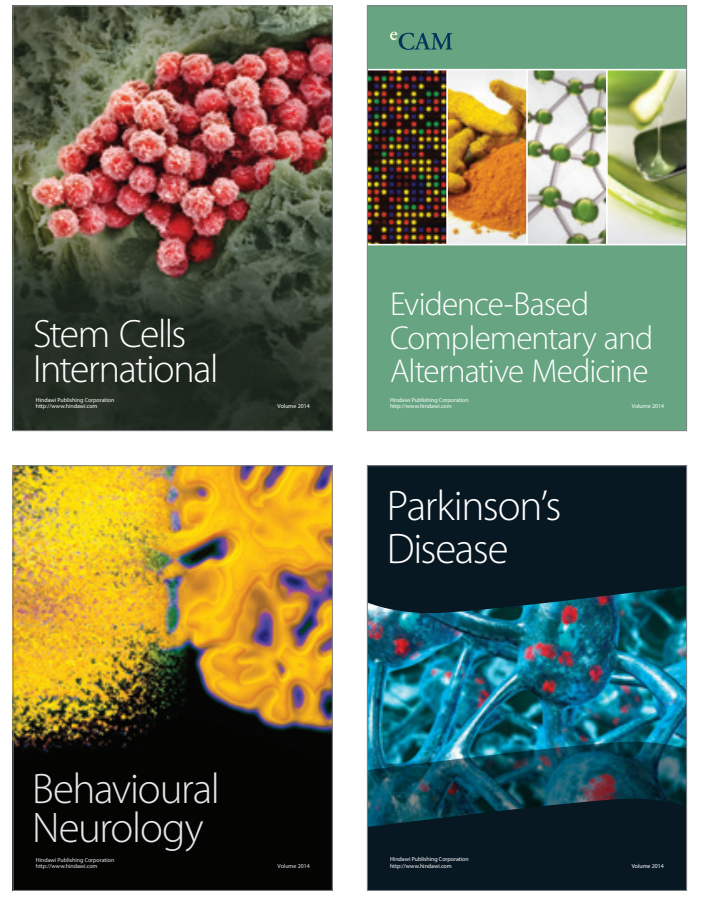
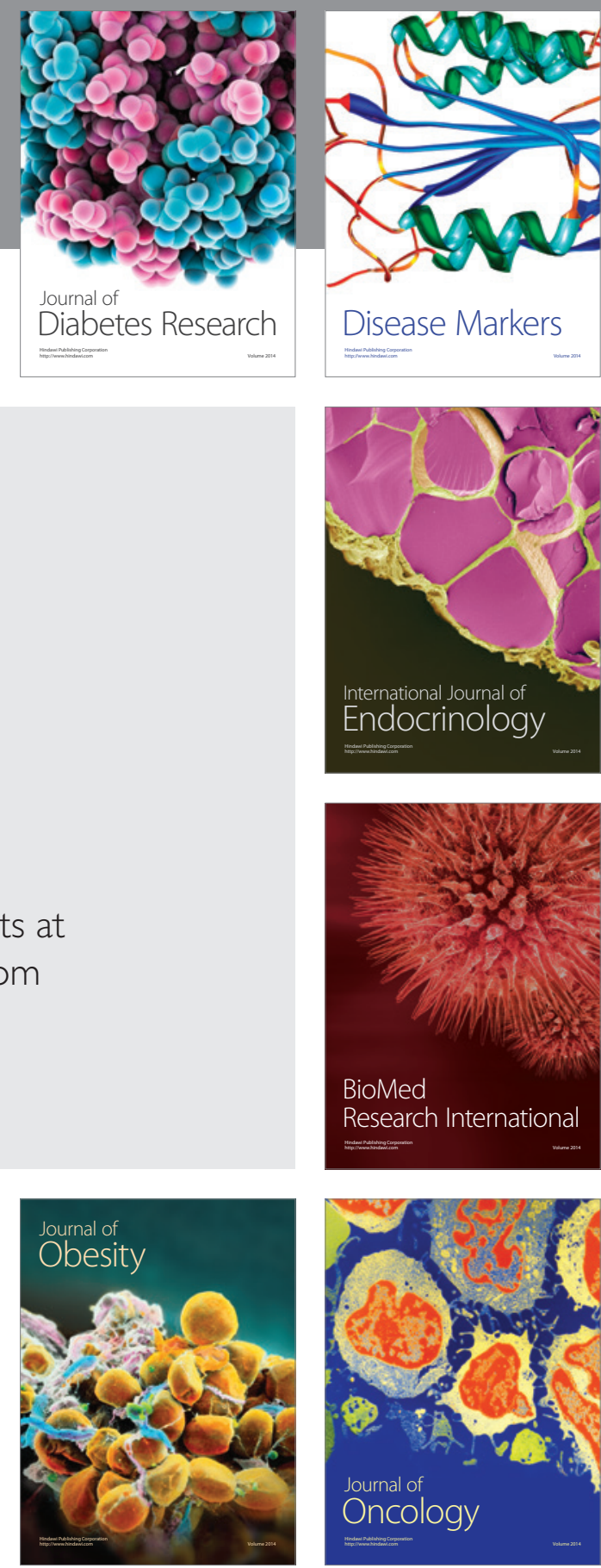

Disease Markers
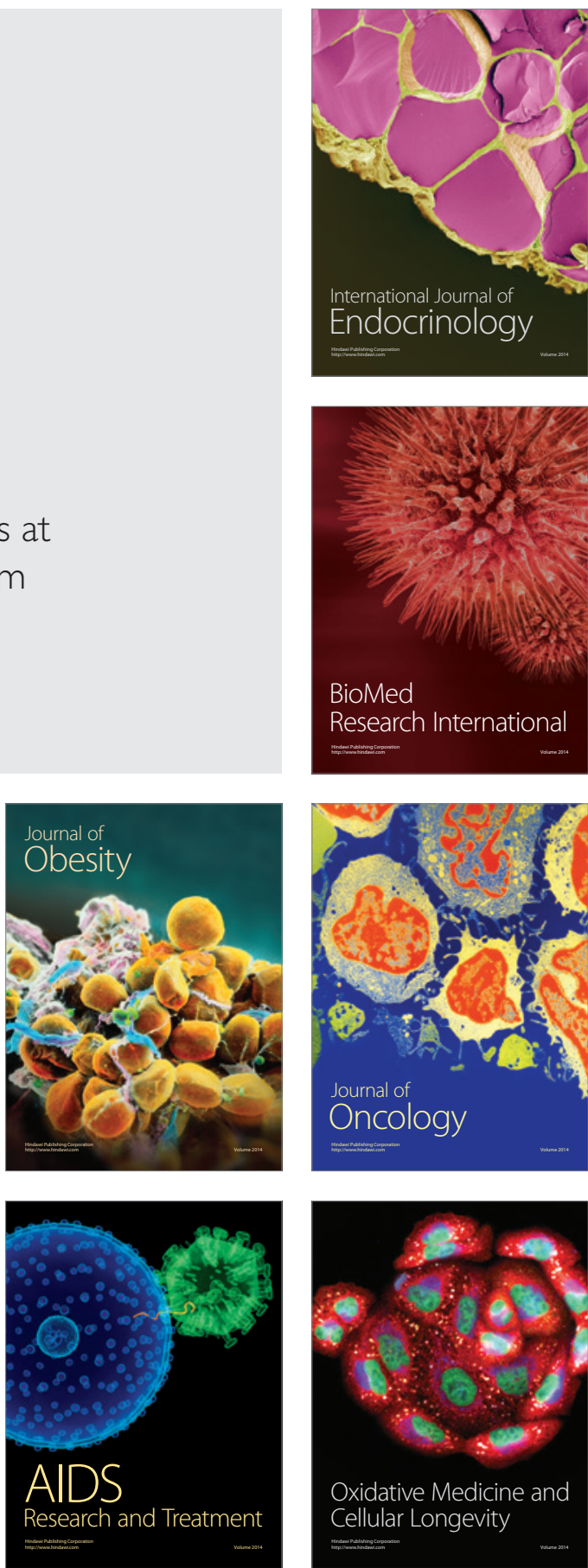